\title{
Quantum-state transfer via resonant tunneling through local-field-induced barriers
}

\author{
S. Lorenzo, ${ }^{1,2}$ T. J. G. Apollaro, ${ }^{1,2,3}$ A. Sindona, ${ }^{1,2}$ and F. Plastina ${ }^{1,2}$ \\ ${ }^{1}$ Dipartimento di Fisica, Università della Calabria, 87036 Arcavacata di Rende (CS), Italy \\ ${ }^{2}$ INFN-Gruppo collegato di Cosenza, 87036 Arcavacata di Rende (CS), Italy \\ ${ }^{3}$ Centre for Theoretical Atomic, Molecular and Optical Physics, School of Mathematics and Physics, \\ Queen's University, Belfast BT7 1NN, United Kingdom \\ (Received 9 January 2013; published 9 April 2013)
}

\begin{abstract}
Efficient quantum-state transfer is achieved in a uniformly coupled spin-1/2 chain, with open boundaries, by application of local magnetic fields on the second and last-but-one spins, respectively. These effective barriers induce the appearance of two eigenstates, bilocalized at the edges of the chain, which allow a high-quality transfer also at relatively long distances. The same mechanism may be used to send an entire e-bit (e.g., an entangled qubit pair) from one to the other end of the chain.
\end{abstract}

DOI: 10.1103/PhysRevA.87.042313

PACS number(s): 03.67.Hk, 75.10.Pq, 03.65.Ud

\section{INTRODUCTION}

Quantum-state transfer (QST), i.e., the reliable transfer of an arbitrary quantum state between different quantum processing units, is one of the major tools of distributed quantum computing and provides the basic "building block" for any quantum communication protocol [1,2]. When the information is encoded in intrinsically localized units, an efficient quantum communication channel can be realized with effective spin systems [3], in order to avoid the difficult problem of interfacing with flying qubits. This channel becomes especially useful for short-ranged, on-chip communication (see Ref. [4], and references therein).

For the QST of one qubit (which may be part of an entangled or, more generally, a correlated pair [5]), a number of protocols have been described employing spin- $\frac{1}{2}$ chains as a quantum data bus to transfer information between their first and last spins (the sender and receiver, respectively). In particular, a high-fidelity transmission can be obtained if additional resources are employed with respect to the original plain scheme of Ref. [3]. Examples include the encoding of quantum states on spatially extended wave packets [6,7], the use of local end-chain operations [8], of local memories and parallel quantum channels [9], or of protocols employing time-dependent interactions [10]. A perfect state transfer, which is unattainable in a uniformly coupled chain, can be achieved instead by a proper preengineering of the coupling strengths. The key advantage in this case is that no external time-dependent controls are needed, as the transfer is realized through the intrinsic dynamics of the chain. Perfect QST, which may be thought of as a particular instance of a more generic swap operation [11], is entailed by accurate settings of the intrachannel coupling strengths giving rise to a linear dispersion relation for excitations propagating across the channel [12]. However, dispersion during transmission occurs in most spin chains due to the nontrivial structure of the many-body Hamiltonian describing the channel, and the design of a nondispersive channel requires a demanding engineering of the Hamiltonian parameters. A systematic analysis on how to set the couplings to allow for a perfect state transfer can be found in Refs. [13,14].
On the other hand, a quasiperfect transfer [15] can be obtained by modifying only a few couplings of an otherwise homogeneous quantum channel [16], in order to obtain a ballistic excitation transfer [17], or Rabi-like oscillations between eigenstates having support only on the sender and receiver sites [18-22].

In this work, we propose a transfer protocol of the latter kind and analyze the efficiency and reliability of state transmission in the presence of a minimal engineering, which depends on the resonant tunneling of spin excitations induced by application of local magnetic fields near the sending and receiving sites. Specifically, we require the sender and receiver to have access and control over the local fields applied on their neighboring spins, which are increased by $\omega$ with respect to the rest of the chain (see the sketch in Fig. 1). As discussed in Refs. [20,21,23], in an open spin- $1 / 2$ chain of $N$ nodes, these extra local fields induce the appearance of two single-particle states, which are "bilocalized" on sites 2 and $N-1$ and can be exploited to perform QST between them $[10,22]$ in a time $t \sim \omega^{N-2}$. However, this is not the only effect produced by the local fields. The geometric confinement, due to the open boundary conditions imposed on the chain, induce the appearance of a further pair of eigenstates which are localized on the first and last sites and can be exploited for a much faster QST. Indeed, once the spin chain is fermionized via the Jordan-Wigner transformation, it is easy to recognize that the local fields create effective potential barriers for the single-particle excitations. If these barriers have equal heights (thus establishing a mirror symmetry [24]), a coherent resonant tunneling occurs between the first and last sites, giving rise to information transfer.

The paper is organized as follows: In Sec II the model with the magnetic field "barriers" is solved, and the appearance of the bilocalized states mentioned above is discussed; in Sec. III the transmission fidelity is studied and the effectiveness of the local fields allowing for a very high-quality QST is demonstrated. Furthermore, in Sec. III B the resilience with respect to noise is analyzed, while in Sec. III C the possibility of transferring more than one qubit is briefly touched upon. After that, in Sec. IV, a time-dependent protocol based on the switching of the local fields is presented and, finally, some concluding remarks are drawn in Sec. V. 


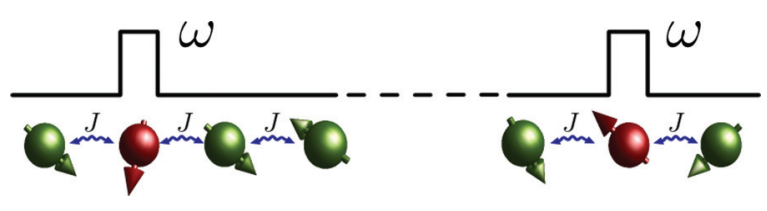

FIG. 1. (Color online) Sketch of the spin chain with sender and receiver located at the first and last sites, and with local field barriers of height $\omega$ applied to the second and last-but-one sites.

\section{THE MODEL AND ITS PROPERTIES}

We consider a linear chain of spin-1/2 particles residing at sites $n=1,2, \ldots, N$ in a lattice of unit lattice constant. The $N$ spins are coupled through the homogeneous nearest-neighbor $X X$ model

$$
\mathcal{H}=-J\left\{\frac{1}{2} \sum_{n=1}^{N-1}\left(\sigma_{n}^{x} \sigma_{n+1}^{x}+\sigma_{n}^{y} \sigma_{n+1}^{y}\right)+\sum_{n=1}^{N} K_{n} \sigma_{n}^{z}\right\},
$$

here expressed in $\hbar=1$ units, which will be used throughout this paper. In Eq. (1), $\sigma_{n}^{\alpha}(\alpha=x, y, z)$ are the usual Pauli matrices for the spin at the $n$th site, probed by a local magnetic field of intensity $K_{n}$, and $J$ is the exchange coupling strength between two nearest-neighboring sites. In the following $J$ will be set to 1 and taken as our energy unit (therefore, times will be given in $1 / \mathrm{J}$ units).

As in the protocol of Ref. [3], we begin with the chain being prepared with all spins up, say, in the initial state $|\mathbf{0}\rangle=|0\rangle^{\otimes N}$ in which $|0\rangle$ and $|1\rangle$ denote the spin-up and -down states along the $z$ axis, respectively. Next, we initialize the first spin of the chain to the state $\left|\psi_{\text {in }}\right\rangle=\alpha|0\rangle+\beta|1\rangle$ and let the chain follow the time evolution generated by the Hamiltonian (1). Since $\left[\mathcal{H}, \sum_{n=1}^{N} \sigma_{z}^{n}\right]=0$, the dynamics take place in the invariant subspaces with 0 and 1 flipped spins, where the former is made up of the state $|\mathbf{0}\rangle$ alone, while the latter is spanned by the computational basis states $|\mathbf{j}\rangle=\sigma_{j}^{+}|\mathbf{0}\rangle \equiv\left|0_{1}, 0_{2}, \ldots, 0_{j-1}, 1_{j}, 0_{j+1}, \ldots\right\rangle$.

The state of the last spin, $\rho_{N}(t)$, is obtained from the time evolved state of the chain by tracing out all but the $N$ th spin, and the aim of the QST protocol is to retrieve the state encoded in the first spin from the last one. The efficiency of the state transfer is then quantified by the fidelity $F(t)=\left\langle\psi_{\text {in }}\left|\rho_{N}(t)\right| \psi_{\text {in }}\right\rangle$, which equals 1 in the case of a perfect transfer. In order to evaluate the channel quality independently of the specific input state, we refer to the average fidelity $\bar{F}(t)$ by integrating $F(t)$ over all possible pure input states of a qubit. This leads to

$$
\bar{F}(t)=\frac{\left|f_{N 1}(t)\right|}{3}+\frac{\left|f_{N 1}(t)\right|^{2}}{6}+\frac{1}{2}
$$

where $f_{N 1}(t)=\left\langle\mathbf{N}\left|e^{-i \mathcal{H} t}\right| \mathbf{1}\right\rangle$ is the transition amplitude of a spin excitation from the first to the last site of the chain. In the following, with the term fidelity we will refer to the quantity given by Eq. (2).

The same effective channel can be used to transfer entanglement, with the first spin sharing an initial singlet state with an external and uncoupled qubit. The amount of (transferred) entanglement between the last spin of the chain and the external one at a subsequent time $t$, as measured by the concurrence, is given by [3]

$$
C(t)=\left|f_{N 1}(t)\right| .
$$

Therefore, in order to perform efficiently both of the tasks, namely, the state and entanglement transfers, it is necessary to achieve a value of $\left|f_{N 1}(t)\right|$ as close as possible to 1 at a certain time $t^{*}$.

Because of the time invariance of the subspaces with a given number of flipped spins, the calculation of $f_{N 1}(t)$ is reduced to diagonalizing the Hamiltonian in the single excitation sector, where Eq. (1) can be expressed as a tridiagonal matrix whose elements are $\mathcal{H}_{n m}^{(1)}=2 K_{n} \delta_{n m}-\left(\delta_{n, n+1}+\delta_{n, n-1}\right)$. Indeed, the transition amplitude $f_{N 1}$ can be written as

$$
f_{N 1}(t)=\sum_{k=1}^{N}\left\langle\mathbf{N} \mid \mathbf{a}_{k}\right\rangle\left\langle\mathbf{a}_{k} \mid \mathbf{1}\right\rangle e^{-i \lambda_{k} t},
$$

where $\lambda_{k}$ are the eigenvalues and $\left|\mathbf{a}_{k}\right\rangle=\sum_{j=1}^{N} a_{k j}|\mathbf{j}\rangle$ are the corresponding eigenvectors of $\mathcal{H}^{(1)}$, arranged in increasing order, i.e., $\lambda_{k^{\prime}}>\lambda_{k}$ for $k^{\prime}>k$.

As we will show below, a large value for $\left|f_{N 1}\right|$ can be obtained by modifying only two local fields in such a way that only two eigenvectors among the $\left|\mathbf{a}_{k}\right\rangle$ 's have a non-negligible superposition with $|\mathbf{1}\rangle$ and $|\mathbf{N}\rangle$. Correspondingly, the time evolution induced by $\mathcal{H}$ gives rise to an effective Rabi oscillation of the spin excitation between the first and the last sites of the chain.

Specifically, we assume that the local magnetic fields are applied to the second and last-but-one spins, which in the following will be denoted as barrier qubits, by setting $K_{n}=\omega\left(\delta_{n, 2}+\delta_{n, N-1}\right)$ in Eq. (1), which gives rise to the model depicted in Fig. 1. This yields an effective decoupling of the first and the last spins of the chain whose dynamics take place mainly in a subspace spanned by two particular eigenstates of $\mathcal{H}^{(1)}$, which are close enough in energy and bilocalized at the edges of the chain.

To confirm these expectations, we study the spectrum of $\mathcal{H}^{(1)}$, reported in Fig. 2 for spin chains of $N=17,18$ sites. The cases of even and odd site numbers are analyzed separately, as they display slightly different features.

In order to quantify the localization of the eigenvectors $\left|\mathbf{a}_{k}\right\rangle$, induced by the magnetic field $\omega$, we use the inverse participation ratio (IPR), whose application to state transfer has been discussed in Ref. [25], which is defined as

$$
\operatorname{IPR}\left(\left|\mathbf{a}_{k}\right\rangle\right)=\frac{\sum_{i=1}^{N}\left|a_{k i}\right|^{2}}{\sum_{i=1}^{N}\left|a_{k i}\right|^{4}} .
$$

When a state is localized on a single site $n$, i.e., $a_{k i}=\delta_{n i}$, the IPR takes its minimum possible value IPR $=1$. On the other hand, an extended state distributed over a large number of sites yields an IPR value of the order of the chain length. Notice that the IPR gives information about the degree of localization of a given eigenstate only, but it does not say anything about its spatial distribution (with the exception of the IPR $=N$ case, corresponding to a state uniformly spread over the whole system).

In Fig. 3, we report the IPR of the eigenstates, ordered by ascending eigenvalues, for $N=17$ and 18 . The effect of increasing $\omega$ is twofold. First, it causes a strong localization of the two eigenvectors $\left|\mathbf{a}_{1,2}\right\rangle: \operatorname{IPR}\left(\left|\mathbf{a}_{1,2}\right\rangle\right) \simeq 2$. These are the 

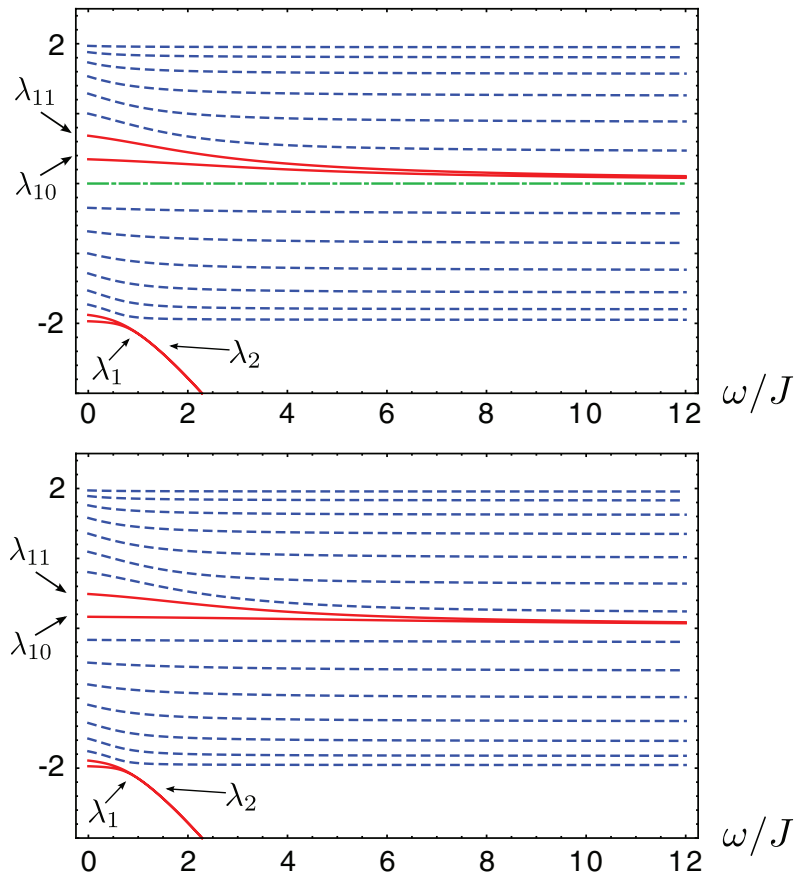

FIG. 2. (Color online) Spectrum of a spin chain with $N=17$ (upper panel) and $N=18$ sites (lower panel) versus $\omega$ : In both cases, the two lowest eigenenergies move outside the band as $\omega$ increases, while the two positive eigenenergies closest to zero become quasidegenerate. The latter are represented by red, solid lines. A zero-energy eigenstate occurs in the odd chain, whose eigenvalue is represented by the green dot-dashed line. All energy values are reported in units of $J$.

two lowest-lying eigenvalues, emerging out the unperturbed $(\omega=0)$ energy band $\lambda_{k} \in(-2,2)$ (see Fig. 2). By increasing $\omega$, these states localize on the two barrier qubits and therefore their contribution to the quantity in Eq. (4) is negligible. Second, another pair of eigenvectors is found, with positive energies close to zero, which reduce their IPR to a value asymptotically tending to 2 for even site numbers [Fig. 3(b)], while remaining slightly above 2 for odd site numbers [Fig. 3(a)].

The localization properties of these eigenstates are crucial for quantum-information transfer as they turn out to give the main contributions in Eq. (4). The remaining intraband eigenstates hold their extended nature and, for even $N$, they have a negligible superposition with the states $\{|\mathbf{1}\rangle,|\mathbf{N}\rangle\}$, so that the dynamics occur in an effective two-level subspace. On the other hand, in the odd- $N$ case, an eigenvector with zero energy eigenvalue is present, which, independently of $\omega$, has a constant amplitude on the sender and receiver sites, given by $\sqrt{\frac{2}{N+1}}$. As a consequence, its contribution to Eq. (4) cannot be neglected for short chains, and the resulting effective dynamics involve three levels. Furthermore, from Fig. 2 we see that other intraband eigenvalues experience a downward shift and the eigenvalues of the bilocalized states become quasidegenerate with energies close to zero.

With these results at hand, we are now in a position to evaluate the transition amplitude $f_{N 1}$, and then the fidelity (2) and the concurrence (3).
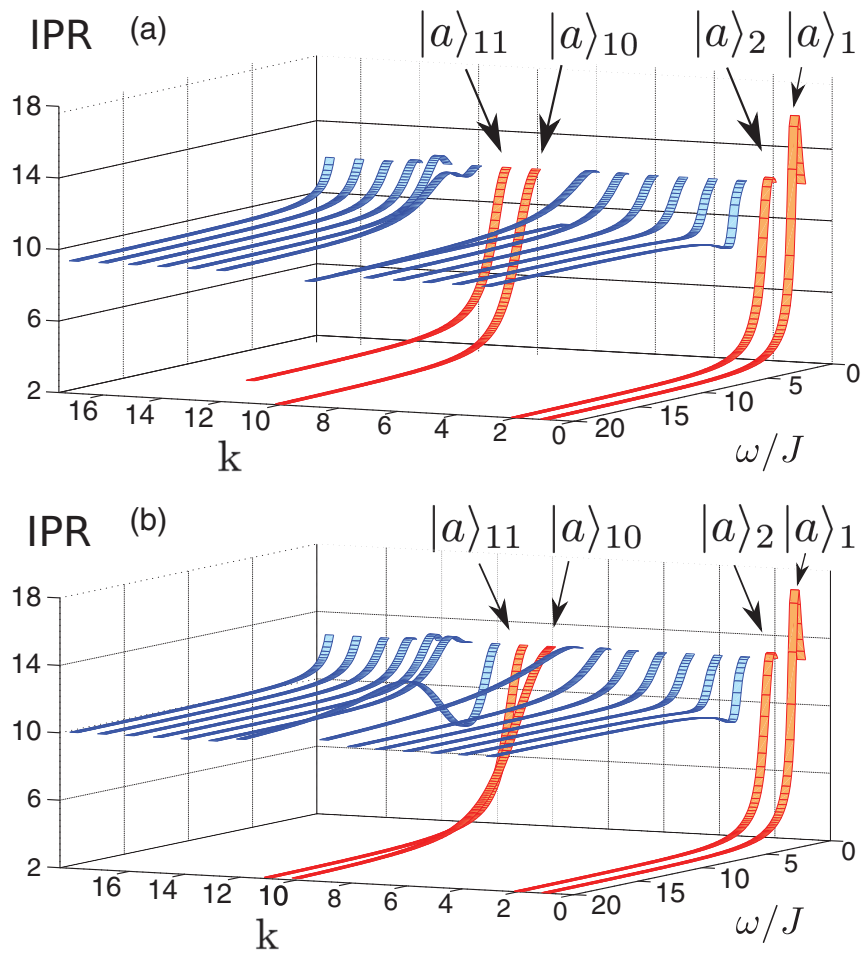

FIG. 3. (Color online) IPR for the eigenstates $|a\rangle_{k}$ of a chain of $N=17$ (upper plot) and $N=18$ (lower plot) sites, sorted by increasing eigenvalues, versus $\omega$ (in units of $J$ ). The first two eigenstates, $|a\rangle_{1}$ and $|a\rangle_{2}$, rapidly reach IPR $\simeq 2$, becoming bilocalized on the barrier qubits. The states corresponding to $k=10,11$, which bilocalize on the sender and receiver qubits, reach the value IPR $\simeq 2$ for the even chain, whereas one of them remains slightly above that value for the odd chain.

\section{FIGURES OF MERIT FOR THE TRANSMISSION}

The average transmission fidelity and the transmitted concurrence are reported in Fig. 4, both as functions of time and chain length, for fixed values of the auxiliary local fields $\omega$ applied to the second and last-but-one sites. To better appreciate the results, they are compared with the homogeneous case $\omega=0$. In Fig. 4(a) we observe a significant

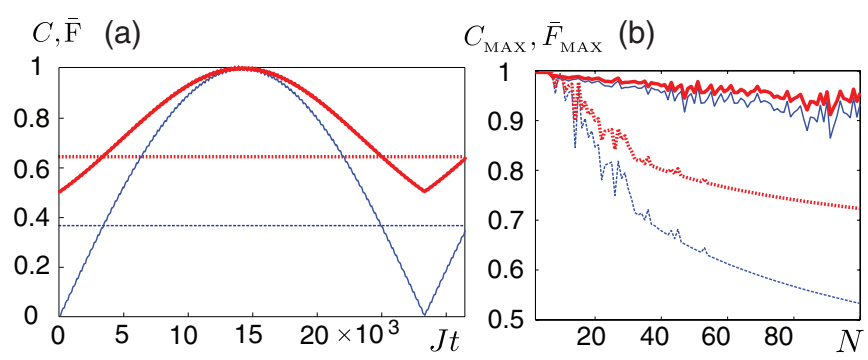

FIG. 4. (Color online) (a) Fidelity (red thick line) and concurrence (blue thin line, starting from $C=0$ at $t=0$ ) for a chain of $N=100$ spins with $\omega=100 \mathrm{~J}$. The dashed red and blue lines are, respectively, the maximum value of the fidelity and of the concurrence attainable for the homogeneous chain with $\omega=0$. Time is expressed in units of $J^{-1}$. (b) Maximum of fidelity (red thick line) and concurrence (blue thin line) versus the number of sites $N$ for $\omega=10 J$ (solid line) and $\omega=0$ (dashed line). 


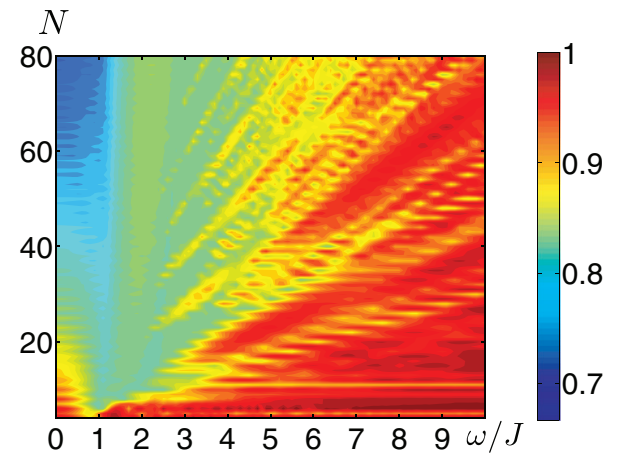

FIG. 5. (Color online) Maximum fidelity in the time interval $J t \in$ $[0,4000]$ as a function of $\omega$ (in units of $J$ ) and $N$. Notice that for $\omega=0$ the fidelity is larger than 0.9 only for short chains, while as $\omega$ increases, the fidelity is significantly enhanced.

improvement of fidelity and concurrence in the presence of $\omega$ with respect to the homogeneous case, while Fig. 4(b) shows that the difference becomes more and more pronounced with increasing the chain length. Indeed, at $\omega=0$, many terms enter the sum (4), giving rise to a destructive interference that rapidly suppresses the transfer efficiency (as measured both by fidelity and concurrence). On the other hand, in the presence of the auxiliary fields $\omega$, only two eigenvectors significantly enter the transition amplitude $f_{N 1}(t)$ so that both the state and entanglement transfers are of high quality.

In Fig. 5 we report the density plot of the maximum fidelity as a function of the number of sites and intensity $\omega$ of the local fields to show that even modest values of $\omega$ are sufficient for high-fidelity state transfer.

By increasing $\omega$, the localization effect is enhanced and, as a result, a better quantum-state transfer is obtained. This is demonstrated in Fig. 6, where the attainable fidelity tends towards 1 both for even and for odd site numbers. Nevertheless, as the eigenvalues of the bilocalized eigenvectors become closer and closer to each other, by increasing $\omega$, the transfer time increases. Since the transfer is based on Rabi-like oscillations between the two eigenvectors with $\mathrm{IPR} \simeq 2$, the transfer time $t_{\text {MAX }}$ can be obtained from their eigenvalues: $t_{\mathrm{MAX}}=\pi /\left(\lambda_{2}-\lambda_{1}\right)$, where $\lambda_{2}>\lambda_{1}$. Furthermore, as shown by a straightforward perturbation analysis, the eigenvalue difference scales as $(N \omega)^{-1}$ for odd site numbers, while it behaves as $\omega^{-2}$ for even ones, resulting in shorter transfer
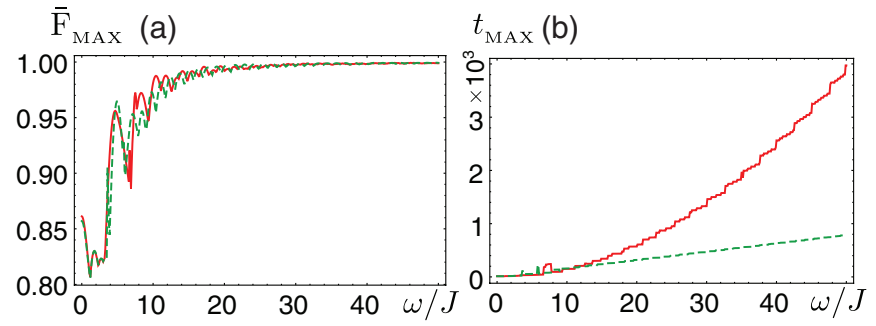

FIG. 6. (Color online) (a) Maximum fidelity achievable in the time interval $J t \in[0,4000]$. (b) Optimal times at which the best transmission is attained. The plots refer to chains of $N=22$ (red solid line) and $N=23$ sites (green dashed line). For odd (even) $N$, $t_{\text {MAX }}$ is linear (quadratic) in $\omega$. times for odd $N$ [see Fig. 6(b)]. Notice that the optimal transfer time does not directly depend on $N$ for even site numbers, but $\omega$ needs to be increased (almost linearly) with increasing $N$ in order to have a fidelity that stays close to unity.

\section{A. Effective Hamiltonian description}

In this section, we compare our results with those obtained using weak-end bonds [18]. To this end, we consider a uniform magnetic field applied to a chain with sender and receiver sites coupled more weakly to their neighboring spins than the other nearest-neighboring sites. Such a week bond is characterized by an interaction strength $J^{\prime}$, being smaller than the intrachain exchange $J$. It turns out that a large fidelity can be obtained provided the ratio $J^{\prime} / J$ is suitably reduced with increasing the chain's length. Moreover, with weak-end bonds, a similar behavior of the transfer time is obtained, with an even-odd asymmetry akin to the one discussed above.

The similarity is explained by observing that the magnetic field barriers on the second and last-but-one spins give rise to effective weak-end bonds, which, however, display some differences with respect to the setup of Ref. [18]. From a perturbation analysis in terms of the small parameter $J / \omega \ll 1$, we infer that the main effect of the local fields is to modify the exchange interaction strengths between pairs of spins near the sender and receiver sites. Indeed, the effective Hamiltonian for the first three spins of the chain reads

$$
H_{\mathrm{eff}}=-\left(\lambda_{+}\left|\psi_{+}\right\rangle\left\langle\mathbf{3}\left|-\lambda_{-}\right| \psi_{-}\right\rangle\langle\mathbf{3}|+\text { H.c. }\right),
$$

where, up to normalization factors, $\left|\psi_{+}\right\rangle \propto \lambda_{-}|\mathbf{1}\rangle+|\mathbf{2}\rangle$, $\left|\psi_{-}\right\rangle \propto \lambda_{+}|\mathbf{1}\rangle+|\mathbf{2}\rangle$, and $\lambda_{ \pm}=\left(\omega \pm \sqrt{\omega^{2}+1}\right)$. In the $\omega / J \rightarrow \infty$ limit, we get $\left|\psi_{+}\right\rangle \rightarrow|\mathbf{2}\rangle$ and $\left|\psi_{-}\right\rangle \rightarrow|\mathbf{1}\rangle$, so that the leading effect of the local fields is the appearance of effective couplings $J_{13}$ and $J_{23}$ between the corresponding spins. The latter are given by

$$
J_{13} \simeq-\frac{1}{2 \omega}, \quad J_{23} \simeq-\frac{1}{2}\left(1-\frac{1}{\omega^{2}}\right) .
$$

Summarizing, the effective Hamiltonian of the first three spins of the chain becomes $H_{\mathrm{eff}}=J_{13}\left(\sigma_{1}^{x} \sigma_{3}^{x}+\sigma_{1}^{y} \sigma_{3}^{y}\right)+$ $J_{23}\left(\sigma_{2}^{x} \sigma_{3}^{x}+\sigma_{2}^{y} \sigma_{3}^{y}\right)+\lambda_{-} \sigma_{1}^{z}+\lambda_{+} \sigma_{2}^{z}$; moreover, due to the presence of the large magnetic field on spin 2, its dynamics is frozen in the $|0\rangle$ state. Similar results hold for the spins near the receiver.

Once the spins at sites $2, N-1$ are adiabatically eliminated, we are effectively left with a chain of $N-2$ spins in a zero magnetic field, uniformly coupled but for the end bonds, where the (effective) couplings between the spins $(1,3)$ and $(N-$ $2, N$ ) have strength $J_{13}$.

A further perturbative analysis in the $J_{13} \ll 1$ limit, performed along the lines of Ref. [18], allow us to write an overall effective Hamiltonian involving the spin-up states at the sending and receiving sites only. More precisely, this is strictly true only if $N$ is even; for a chain with an odd number of sites, instead, the inclusion of an auxiliary state is necessary, corresponding to the zero-energy eigenstate, whose effects have been discussed in Sec. II.

As a result, for $N$ even and odd, respectively, the state transfer is described by the following effective 


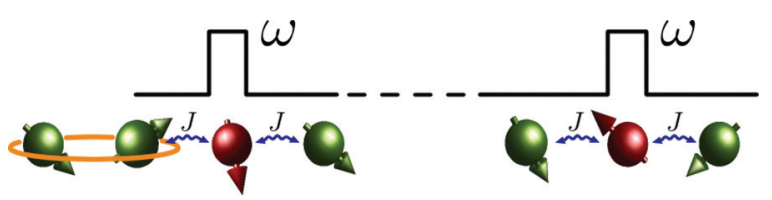

FIG. 7. (Color online) Scheme of the setup used for entanglement transfer from the qubit pair $(0,1)$ to the pair $(0, N)$, where the qubit 0 is decoupled from the chain.

Hamiltonians:

$$
\begin{gathered}
H_{\mathrm{eff}}^{\mathrm{even}}=-\left(\frac{1}{4 \omega^{2}}|\mathbf{1}\rangle\langle\mathbf{N}|+\text { H.c. }\right), \\
H_{\mathrm{eff}}^{\text {odd }}=\frac{1}{2 \omega}\left(1-\frac{4}{N-3}\right)(|\mathbf{1}\rangle\langle\mathbf{1}|+| \mathbf{N}\rangle\langle\mathbf{N}|) \\
-\sqrt{\frac{1}{2(N-3)}} \frac{1}{\omega}\left(|\mathbf{1}\rangle\left\langle\left. a\right|_{(N+1) / 2}+\mid \mathbf{N}\right\rangle\left\langle\left. a\right|_{(N+1) / 2}+\text { H.c. }\right) .\right.
\end{gathered}
$$

\section{B. Robustness against noise}

In this section we investigate how a static disorder in the magnetic fields acting on the qubits $n=3, \ldots, N-2$ affects the efficiency of information transfer, and in particular, to be specific, of entanglement transfer, performed according to the scheme depicted in Fig. 7. In this setting the entanglement, initially contained in the state $\left|\Psi_{+}\right\rangle=\frac{1}{\sqrt{2}}(|01\rangle+|10\rangle)$ of the qubit pair $(0,1)$, is transferred to the pair $(0, N)$ and is quantified by the concurrence as given by Eq. (3).

The kind of disorder we consider is given by the presence of random local magnetic fields between the barrier qubits. In other words, we are assuming that local random magnetic fields, uniformly distributed in an interval $-b<K_{n}<b$, with $b$ denoting the disorder strength, act on the spins residing on sites $n=3, \ldots, N-2$. This choice is justified by the fact that the Hamiltonian parameter of the qubits $(1, N)$ are generally considered to be more precisely controllable in order to efficiently perform the state encoding and readout procedure, and therefore, they will be practically unaffected by the disorder. Furthermore, we allow the same degree of control for the neighboring spins $2, N-1$, whose local fields are assumed to be precisely fixed. In Fig. 8, we see that the attainable concurrence (3), averaged over $10^{5}$ samples of disorder, remains quite high provided that $b \ll \omega$. Indeed, the bilocalized nature of the relevant eigenstates is not significantly perturbed. On the contrary, this is no longer the case for values of $b$ comparable to-or greater than- $\omega$. Similar results are obtained for the fidelity of the QST.

Depending on the specific physical implementation of the model, other sources of errors (and, specifically, of static disorder) can be identified. In particular, we would like to mention that the robustness of different transfer schemes against bond disorder (that is, static disorder in the spincoupling strengths) has been investigated in Ref. [26]. It turns out that the localization properties of the eigenstates play an important role for efficient state transfer in the presence of nonuniform bonds, and that a mechanism based on localized states, like the one we are describing, is more resilient than
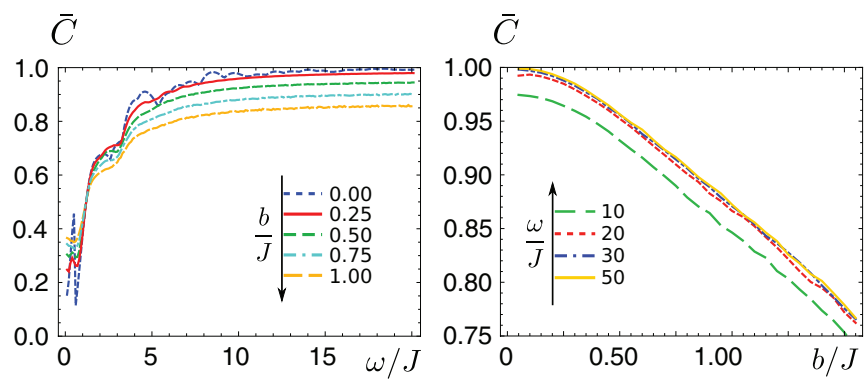

FIG. 8. (Color online) (Left panel) Averaged concurrence vs auxiliary field strength $\omega$ for different values of the disorder parameter $b$. (Right panel) Concurrence vs disorder strength $b$ for different values of $\omega$. The curves for $\omega>20$ are almost indistinguishable and no relevant change in the effects of the disorder is observed. In both panels, the length of the chain is $N=10$ and averages are performed over $10^{5}$ realizations of disorder. All energy values are expressed in units of $J$.

a ballistic transport-based one. On the other hand, since we consider high magnetic field applied locally to sites 2 and $N-1$, a leakage effect is certainly possible, affecting the neighboring sites. To check the robustness of our transfer scheme against this lack of control, we can consider random magnetic fields, with amplitude decaying with the distance, to affect the dynamics of spins $3,4, N-3$, and $N-2$ (on the other hand, as discussed above, we assume a very high degree of control on the sending and receiving sites, and on the barrier fields). The results of such an analysis are reported in Fig. 9, where the transmission fidelity averaged over $10^{5}$ realization of these static random fields is displayed. For very small values of the local fields $\omega$, the quality of the transfer is strongly reduced by the presence of this kind of disorder, while its effect is shown to substantially decrease for larger values of barrier fields, despite the fact that residual static random fields are always bounded by the same fractions of $\omega$.

The plots suggest that, both for chains with odd and even $N$, an optimal value of the local barrier fields exists in the case in which a given fraction of it is assumed to leak to the neighboring sites. If such an optimal value of $\omega$ is selected
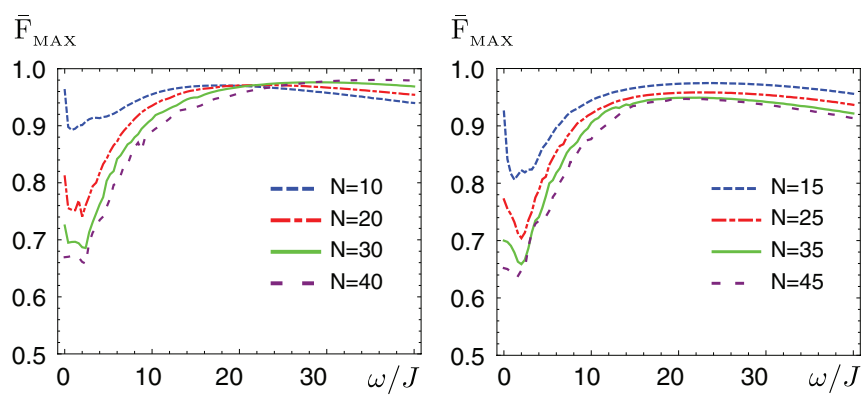

FIG. 9. (Color online) Averaged fidelity vs auxiliary field strength $\omega$ for different lengths of the chain. In this case a residual magnetic field is supposed to act on sites $3, N-2$ and $4, N-3$, with random values uniformly distributed between 0 and $\omega / 10$ for the sites near the barriers, and between 0 and $\omega / 40$ for the next to nearest sites, respectively. The plots show averages performed over $10^{5}$ realizations. 


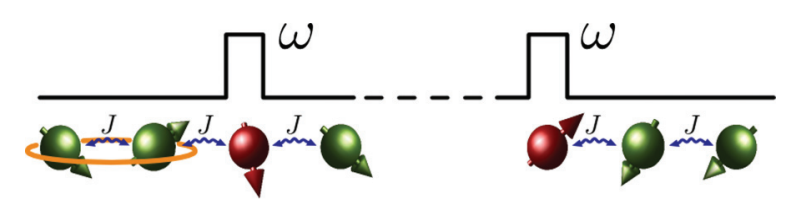

FIG. 10. (Color online) Sketch of the configuration for the transfer of an e-bit.

(which scales almost linearly with the size $N$ ), the average fidelity is kept very close to unity.

\section{Transport of an entire e-bit}

We have shown above that a qubit encoded on the first spin of the chain is almost perfectly transferred to the other end, thanks to the application of local magnetic fields to the adjacent spins to the sender and the receiver sites. In this section we extend this idea to the transfer of an entangled pair. Considering the setup depicted in Fig. 10, we aim at transferring the entanglement shared by qubits 1 and 2 to qubits $N-1$ and $N$ by use of auxiliary magnetic fields applied to sites 3 and $N-3$. We thus allow $K_{n}=\omega\left(\delta_{n, 3}+\delta_{n, N-2}\right)$ in Eq. (1). Then, we start from the fully polarized state $|\mathbf{0}\rangle$, and initialize the first two spins in a state belonging to the single excitation subspace so that the initial state of the whole chain reads

$$
|\Psi(0)\rangle=\alpha|\mathbf{1}\rangle+\beta|\mathbf{2}\rangle, \quad\left(|\alpha|^{2}+|\beta|^{2}\right)=1,
$$

whose evolution is given by

$$
|\Psi(t)\rangle=\sum_{j=1}^{N} p_{j}|\mathbf{j}\rangle, \quad p_{j}=\alpha\left\langle\mathbf{j}\left|e^{-i H t}\right| \mathbf{1}\right\rangle+\beta\left\langle\mathbf{j}\left|e^{-i H t}\right| \mathbf{2}\right\rangle .
$$

Finally, we obtain the state of the qubits $N-1$ and $N$ by performing the partial trace over the first $N-2$ spins. Considering an initially entangled $(1,2)$ pair (that is, $\alpha, \beta \neq 0$ ), the amount of entanglement transferred to the pair $(N-1, N)$ and measured by the concurrence is given by $C_{N-1, N}=$ $2\left|p_{N-1} p_{N}\right|$. As shown in Fig. 11 where an initial maximally entangled state has been taken, i.e., $\alpha=\beta=\frac{1}{\sqrt{2}}$, also the entanglement may be efficiently transferred in the presence of the auxiliary magnetic fields.

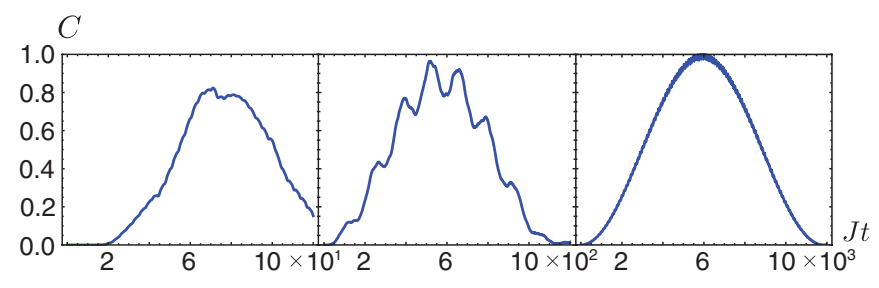

FIG. 11. (Color online) Concurrence transferred from the maximally initial entangled qubits pair $(1,2)$ to the pair $(N-1, N)$ for a chain of $N=33$ and $\omega=5,15,45$ (from left to right). Higher values of the local field, besides increasing the amount of transferred entanglement, regularize the dynamics.

\section{TIME-DEPENDENT QUANTUM-STATE TRANSFER PROTOCOL}

In this section we investigate a QST protocol in which we allow for time control of the magnetic fields acting on the barrier qubits. The aim of this control is to provide a precise timing for the beginning and end of the sending stage, as given by the switching of the local fields. At the same time, the control relaxes the need of a fast (in fact, instantaneous) extraction of the received information at the site $N$ once the transmission is performed. The idea is to encode the quantum state on the sender site and leave it there for a future transmission by means of a strong magnetic field on its neighbor barrier qubits. In this first step the information stays localized on the sender as no tunneling of the spin excitation is possible due to the energy mismatch with other sites. The sending stage is then realized by switching on the magnetic field of the other barrier, at the $(N-1)$ th site. During this second step, the Rabi oscillation described in the previous sections takes place. Finally, in the third stage, only the barrier on the last-but-one spin is left on, in order to trap the received quantum state, while the local field near the sender site is switched off.

To implement this proposal, we exploit the time-dependent Hamiltonian

$\mathcal{H}(t)=-\frac{1}{2} \sum_{n=1}^{N-1}\left(\sigma_{n}^{x} \sigma_{n+1}^{x}+\sigma_{n}^{y} \sigma_{n+1}^{y}\right)-\sum_{i=2, N-1} \omega_{i}(t) \sigma_{i}^{z}$,

where

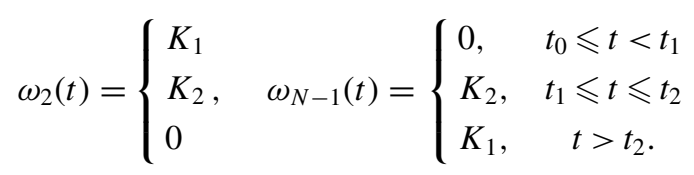

Here, $t_{2}=t_{1}+\Delta t$, with $\Delta t$ being an optimal transfer time interval, which we define below. With this time-dependent field configuration, the spin at the first site is "frozen" until $t<t_{1}$ as the state $|\mathbf{1}\rangle$ is an approximate eigenstate of $\mathcal{H}\left(t<t_{1}\right)$; then, after the resonant tunneling to the receiving site $t_{1} \leqslant t \leqslant$ $t_{1}+\Delta t$, for $t>t_{2}$, the information is definitely stored in the $N$ th spin, as $|\mathbf{N}\rangle$ is an approximate eigenstate of $\mathcal{H}\left(t>t_{2}\right)$ (see the upper panel in Fig. 12).

Since the transition amplitude is given by $\langle N|U(t)| 1\rangle$, in order to obtain the fidelity, one needs to solve the time-dependent Schrödinger equation for the state $U(t)|1\rangle=\sum_{k=1}^{N} \beta_{k}(t)|k\rangle$ that reduces to an $N \times N$ system of differential equations:

$$
\begin{aligned}
& i \frac{d \beta_{1}(t)}{d t}=-\beta_{2}(t), \\
& i \frac{d \beta_{2}(t)}{d t}=-\beta_{1}(t)-\omega_{2}(t) \beta_{2}(t)-\beta_{3}(t), \\
& \ldots \\
& i \frac{d \beta_{j}(t)}{d t}=-\beta_{j-1}(t)-\beta_{j+1}(t) \quad(j=3, \ldots, N-2), \\
& \ldots \\
& i \frac{d \beta_{N-1}(t)}{d t}=-\beta_{N-2}(t)-\omega_{N-1}(t) \beta_{N-1}(t)-\beta_{N}(t), \\
& i \frac{d \beta_{N}(t)}{d t}=-\beta_{N-1}(t) .
\end{aligned}
$$



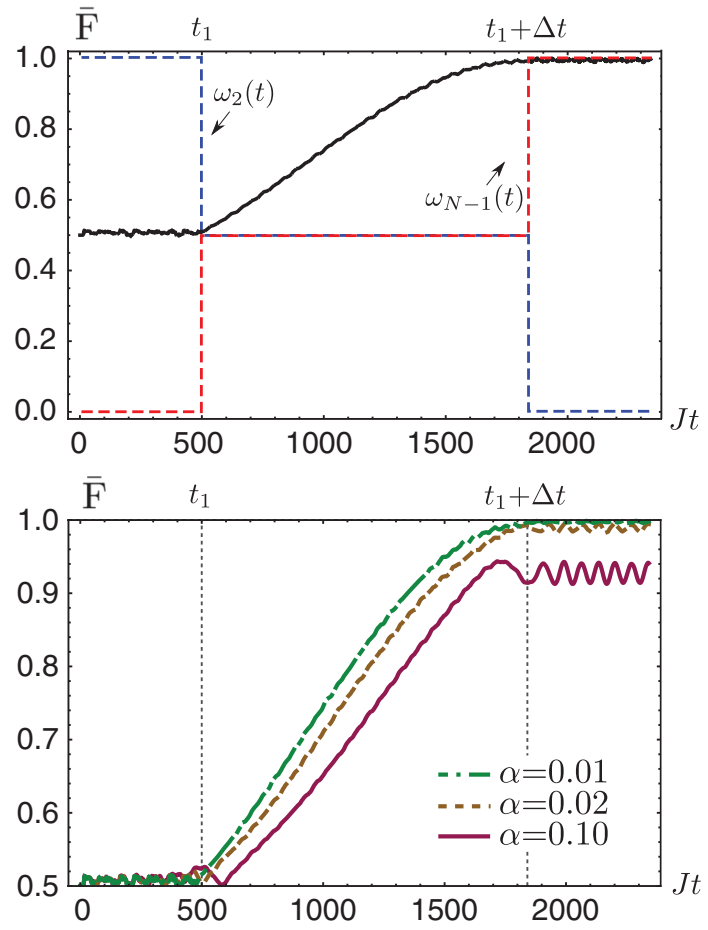

FIG. 12. (Color online) (Upper panel) Average fidelity for a chain of $N=30$ sites with $K_{1}=60$ and $K_{2}=30$, where the three steps of the QST protocol are clearly visible from the time behavior of the two fields, which are switched according to the recipe of Eq. (11). (Lower panel) Same as in the upper plot, but with finite switching times for the fields. In this case, $\omega_{2}(t)$ and $\omega_{N-1}(t)$ are smoother versions of the step functions of Eq. (11), with exponential corrections: $\omega_{2}(t)=K_{2} /\left[\exp \left\{\alpha\left(t-t_{2}\right)\right\}+1\right]+\left(K_{1}-\right.$ $\left.K_{2}\right) /\left[\exp \left\{\alpha\left(t-t_{1}\right)\right\}+1\right]$, and a similar behavior for $\omega_{N-1}(t)$. The three curves correspond to three values of $\alpha$ and it turns out that the achievable $\bar{F}$ decreases with the steps becoming smoother and smoother (that is, with increasing $\alpha$ ).

The solution, in each time interval where $\omega_{2}(t)$ and $\omega_{N-1}(t)$ are constant, is

$$
\beta_{s}\left(t_{i+1}\right)=\left.(-1)^{s+1} \sum_{\lambda_{j}} \sum_{k=1}^{N} \frac{Q_{k, s}(\lambda)}{\frac{d P(\lambda)}{d \lambda}}\right|_{\lambda_{j}} e^{-i \lambda_{j} t} \beta_{k}\left(t_{i}\right),
$$

where $\lambda_{j}$ are the eigenvalues of $H\left(t_{i}\right)$, while $P(\lambda)$ and $Q_{k, j}(\lambda)$ are, respectively, the determinant and the minors of the matrix $\left[H\left(t_{i+1}\right)-\lambda \mathbb{1 1}\right]$. In order to perform the state transfer along the chain, the optimal time $\Delta t$ is proportional, again, to the inverse of the eigenvalue difference of the intermediate stage Hamiltonian, and reads

$$
\Delta t= \begin{cases}\frac{\pi}{2} K_{2}^{2}, & N \text { even } \\ \frac{\pi}{4}(N-3) K_{2}, & N \text { odd } .\end{cases}
$$

The procedure works quite well for even $N$, as illustrated in Fig. 12, where the detrimental effect of finite switching times for the field is also explored. Unlike adiabatic transfer schemes [27], here a fast switching of the magnetic fields is desirable because the overlap of the initial (final) state $|\mathbf{1}\rangle(|\mathbf{N}\rangle)$ with the bilocalized states is maximized by a step function, whereas a smoother switching function would introduce into the dynamics destructively interfering states that do not possess the required localization properties. This is illustrated in the lower panel of Fig. 12, where the average fidelity is plotted for different switching rates.

For odd chains, on the other hand, the presence of the zero-energy eigenstate taking part in the dynamics, makes the state transfer more involved, because the trapping stages both at the beginning and end of the protocol are not so efficient.

\section{CONCLUSIONS}

Spin chain models describe a great variety of different physical systems, ranging from trapped ions interacting with lasers [28], via flux qubits [29] and arrays of coupled cavity, to ultracold atoms in one-dimensional optical lattices [30,31], including coupled quantum dots [32], nitrogen vacancy centers in diamond [33], or magnetic molecules [34]. All these possible implementations have their own strengths and weaknesses and allow for different possible kinds of controls on the single units. It is therefore of interest to put forward QST protocols that may fit better to a specific experimental realization of the quantum channel.

In many of the above-mentioned implementations, only a restricted access is possible to the Hamiltonian parameters. It is therefore desirable, to study efficient and reliable transmission protocols that require only a limited amount of controls. In this paper we have shown that a high-quality quantum-state transfer can be achieved in an $X X$-spin chain by means of strong local magnetic fields applied on the second and last-but-one spins, that cause the appearance of two specific eigenstates, bilocalized on the sender and receiver sites located at the edges of the chain. Unlike other QST protocols, this implies that no engineering of the Hamiltonian parameters is required. A much more limited control is needed only on some local properties of the spins close to the sender and the receiver sites. By increasing the magnetic fields $\omega$, the transfer fidelity has been shown to approach unity, with a transfer time scaling as $\omega^{-1}$ and $\omega^{-2}$ for chains with odd and even numbers of sites, respectively; furthermore, a good resilience to the presence of static disorder in the local Hamiltonian parameters of the channel has been demonstrated. The model works also for the transfer of a two-qubit state, and more general $n$-qubit state transmission can be easily envisaged using similar schemes. Furthermore, this setup allows for an efficient time-dependent protocol, based on fast switching of the magnetic fields, which has the benefit of avoiding the need for a fast and well synchronized state retrieval. The latter is a common requirement for many existing QST proposals. Indeed, with our setup, the transferred state can be trapped, with a high fidelity of storage, at the end of the transmission protocol, thus allowing for a much easier extraction of the information.

\section{ACKNOWLEDGMENTS}

T.J.G.A. is supported by the European Commission, the European Social Fund, and the Region Calabria through the program POR Calabria FSE 2007-2013-Asse IV Capitale Umano-Obiettivo Operativo M2. 
[1] M. A. Nielsen and I. L. Chuang, Quantum Computation and Quantum Information (Cambridge University Press, Cambridge, UK, 2000); V. Vedral, Introduction to Quantum Information Science (Oxford University Press, New York, 2007).

[2] H. J. Kimble, Nature (London) 453, 1023 (2008).

[3] S. Bose, Phys. Rev. Lett. 91, 207901 (2003).

[4] S. Bose, Contemp. Phys. 48, 13 (2007); D. Burgarth, Eur. Phys. J. Spec. Top. 151, 147 (2007); T. J. G. Apollaro, S. Lorenzo, and F. Plastina, Int. J. Mod. Phys. B 27, 1345035 (2013).

[5] S. Campbell, T. J. G. Apollaro, C. DiFranco, L. Banchi, A. Cuccoli, R. Vaia, F. Plastina, and M. Paternostro, Phys. Rev. A 84, 052316 (2011).

[6] S. Paganelli, G. L. Giorgi, and F. de Pasquale, Fortschr. Phys. 57, 1094 (2009).

[7] T. J. Osborne and N. Linden, Phys. Rev. A 69, 052315 (2004).

[8] C. Di Franco, M. Paternostro, and M. S. Kim, Phys. Rev. Lett. 101, 230502 (2008).

[9] V. Giovannetti and D. Burgarth, Phys. Rev. Lett. 96, 030501 (2006); D. Burgarth and S. Bose, New J. Phys. 7, 135 (2005); Phys. Rev. A 71, 052315 (2005); A. Bayat, S. Bose, and P. Sodano, Phys. Rev. Lett. 105, 187204 (2010).

[10] H. L. Haselgrove, Phys. Rev. A 72, 062326 (2005); D. Zueco, F. Galve, S. Kohler, and P. Hänggi, ibid. 80, 042303 (2009); R. Heule, C. Bruder, D. Burgarth, and V. M. Stojanovic, ibid. 82, 052333 (2010).

[11] B. Q. Liu, L. A. Wu, B. Shao, and J. Zou, Phys. Rev. A 85, 042328 (2012).

[12] M. Christandl, N. Datta, A. Ekert, and A. J. Landahl, Phys. Rev. Lett. 92, 187902 (2004).

[13] L. Vinet and A. Zhedanov, Phys. Rev. A 85, 012323 (2012).

[14] M. Bruderer, K. Franke, S. Ragg, W. Belzig, and D. Obreschkow, Phys. Rev. A 85, 022312 (2012).

[15] L. Vinet and A. Zhedanov, Phys. Rev. A 86, 052319 (2012).

[16] M. J. Hartmann, M. E. Reuter, and M. B. Plenio, New J. Phys. 8, 94 (2006); L. Campos Venuti, C. Degli Esposti Boschi, and M. Roncaglia, Phys. Rev. Lett. 99, 060401 (2007); L. Campos Venuti, S. M. Giampaolo, F. Illuminati, and P. Zanardi, Phys. Rev. A 76, 052328 (2007); G. Gualdi, S. M. Giampaolo, and F. Illuminati, Phys. Rev. Lett. 106, 050501 (2011); L. Banchi, A. Bayat, P. Verrucchi, and S. Bose, ibid. 106, 140501 (2011).

[17] L. Banchi, T. J. G. Apollaro, A. Cuccoli, R. Vaia, and P. Verrucchi, Phys. Rev. A 82, 052321 (2010); T. J. G. Apollaro, L. Banchi, A. Cuccoli, R. Vaia, and P. Verrucchi, ibid. 85, 052319 (2012).
[18] A. Wójcik, T. Łuczak, P. Kurzyński, A. Grudka, T. Gdala, and M. Bednarska, Phys. Rev. A 72, 034303 (2005); 75, 022330 (2007).

[19] G. Gualdi, V. Kostak, I. Marzoli, and P. Tombesi, Phys. Rev. A 78, 022325 (2008).

[20] F. Plastina and T. J. G. Apollaro, Phys. Rev. Lett. 99, 177210 (2007).

[21] S. Paganelli, F. de Pasquale, and G. L. Giorgi, Phys. Rev. A 74, 012316 (2006).

[22] A. Casaccino, S. Lloyd, S. Mancini, and S. Severini, Int. J. Quantum. Inf. 7, 1417 (2009); T. Linneweber, J. Stolze, and G. S. Uhrig, ibid. 10, 1250029 (2012).

[23] T. J. G. Apollaro and F. Plastina, Phys. Rev. A 74, 062316 (2006).

[24] T. Shi, Y. Li, Z. Song, and C. P. Sun, Phys. Rev. A 71, 032309 (2005); P. Karbach and J. Stolze, ibid. 72, 030301(R) (2005).

[25] A. Zwick and O. Osenda, J. Phys. A: Math. Theor. 44, 105302 (2011).

[26] A. Zwick, G. A. Alvarez, J. Stolze, and O. Osenda, Phys. Rev. A 84, 022311 (2011); 85, 012318 (2012); D. Petrosyan, G. M. Nikolopoulos, and P. Lambropoulos, ibid. 81, 042307 (2010).

[27] B. Chen, W. Fan, Y. Xu, Z. Y. Chen, X. L. Feng, and C. H. Oh, Phys. Rev. A 86, 012302 (2012).

[28] D. Porras and J. I. Cirac, Phys. Rev. Lett. 92, 207901 (2004).

[29] A. Romito, R. Fazio, and C. Bruder, Phys. Rev. B 71, 100501(R) (2005); F. W. Strauch and C. J. Williams, ibid. 78, 094516 (2008); D. I. Tsomokos, M. J. Hartmann, S. F. Huelga, and M. B. Plenio, New J. Phys. 9, 79 (2007).

[30] B. P. Lanyon et al., Science 334, 57 (2011); J. F. Sherson, C. Weitenberg, M. Endres, M. Cheneau, I. Bloch, and S. Kuhr, Nature 467, 68 (2010); S. M. Giampaolo and F. Illuminati, Phys. Rev. A 80, 050301(R) (2009); New J. Phys. 12, 025019 (2010).

[31] U. Dorner, P. Fedichev, D. Jaksch, M. Lewenstein, and P. Zoller, Phys. Rev. Lett. 91, 073601 (2003); L.-M. Duan, E. Demler, and M. D. Lukin, ibid. 91, 090402 (2003); M. J. Hartmann, F. G. S. L. Brandao, and M. B. Plenio, ibid. 99, 160501 (2007); S. Yang, A. Bayat, and S. Bose, Phys. Rev. A 84, 020302(R) (2011).

[32] G. M. Nikolopoulos, D. Petrosyan, and P. Lambropoulos, Europhys. Lett. 65, 297 (2004); D. Petrosyan and P. Lambropoulos, Opt. Commun. 264, 419 (2006).

[33] N. Y. Yao, L. Jiang, A. V. Gorshkov, Z.-X. Gong, A. Zhai, L.-M. Duan, and M. D. Lukin, Phys. Rev. Lett. 106, 040505 (2011).

[34] G. A. Timco et al., Nat. Nanotechnol. 4, 173 (2009). 